\title{
Toward Opportunities for Regional Collaborations in Drug Discovery in the Midwest
}

\author{
R. V. Duncan
}

Vice Chancellor for Research, University of Missouri

$\mathrm{M}$ idwest universities have become research powerhouses in their own right, but large centralized investments to enable competitive positions in capital generally at larger universities that exist typically along the coasts. Here we propose a new regional collaboration in cloud bio-computing, shared core facility support, transgenic animal model development, and clinical trials that will compete successfully with other drug discovery activities elsewhere in the United States, and throughout the world. The west-central region of Missouri and the eastern region of Kansas have successfully established themselves internationally through regional development non-profits as the world's 'Animal Health Corridor'. Here we propose to follow a similar international marketing strategy to gain recognition as a center for human health research and drug discovery.

This will become possible only when truly comprehensive and competitive capabilities have been established through these essential collaborative agreements between major Midwest 'flagship' research universities. It is essential that this effort to secure major drug development deals does not go forward until all capabilities have been established to make a clearly competitive value proposition to the pharmaceutical industry.

\section{Introduction}

Over the last several decades the University of Missouri (MU) has invested extensively in the basic biosciences, in comparative medicine, and in transgenic technologies for research animal model development.
Over this same period the University of Kansas (KU) has become very strong in clinical trial development for pharmaceuticals. This naturally leads to an opportunity to build on each institution's strengths collaboratively as we build a world-leading capability in bio-innovation and pharmaceutical development in human health care. Many other institutions in the greater Kansas City area, including UMKC, the major hospitals, and the Kansas City University of Medicine and Bioscience (KCUMB), and for-profit firms, all offer specific expertise that will greatly enable the ability within the Greater Kansas City Region to compete with other bioinnovation centers around the world. Children's Mercy Hospital has 
established itself as a center for personal medicine, and St. Luke's Hospital is renowned for their world-class program in stroke prevention and recovery, to include the development of highly innovative catheter systems to remove threatening blood clots from the circulatory system near the base of the brain. KCUMB currently trains more licensed physicians than any other educational institution in Missouri, and they have developed optimized custom facilities to support human medical trials. These outstanding capabilities, coupled with the emergence of a new emphasis on regionalism that is discussed in detail below, promise to create the environment necessary for this region to emerge as the point of choice for drug and human health care development world-wide within the next five years. This, coupled with the expanded regional marketing ability of the Kansas City Area Development Council (KCADC), which successfully branded this region as the Animal Health Corridor world-wide, all indicate that this success is eminently achievable once the regional development has expanded to assure a competitive value proposition in drug discovery to the pharmaceutical industry.

The essential aspect of this regional emergence is that all partners remain focused on the outcome of such a successful collaboration. The issue must never become 'who is getting more than whom' in a particular drug development deal, but rather the collective best allocation of regional resources to outperform competition along the Coasts. This will require complete transparency in a non-profit organization with even and equal representation from all participatory institutions. This nonprofit would transparently solicit, structure, and propose the parsing of these major deals based on proven ability to perform. It is the ability to represent the collective capabilities of all institutions concerned that will make the Midwest Region more attractive than existing drug discovery operations elsewhere in the United States. We at MU have been approached by numerous international investment groups that are interested in developing a high-tech based drug development effort on the MU campus. Such regional efforts will succeed over the competition if the complementary strengths across the Midwest Region are effectively brought together in a single, external marketing operation. Nothing will prevent each institution's efforts to solicit other business alone simultaneously, but all institutions will need to agree not to interfere or compete with a particular deal that is being negotiated by the nonprofit on behalf of all institutions.

\section{Regional Cloud Computing}

The KCALSI has worked closely with many Chief Information Officers from institutions within the Kansas City Area to propose a mutual investment in cloud bio-computing, which would meet a major need within the Region for drug discovery, and other applications. This effort, which has been led by Dr. Gary Allen, CIO of MU, will soon propose a structure for such a shared investment across the Midwest Region. Other areas of the United States have found that such cooperative investments achieve a better economy of scale, and we anticipate that this will be realized in the 
Great Plains Network as well. IBM has expressed interest in this, and MU has recently won a SUR (Shared University Research) Award to develop cloud computing. Further, I have joined with the MU Development staff and some of our lead research faculty members in a detailed meeting with Mark Dean, Vice President of IBM, and we have agreed that the development of cloud biocomputing is an important shared goal of both IBM and MU. Following this meeting and after further discussions with those mentioned above, MU's CIO Gary Allen, has structured and led a group of CIOs from the University of Kansas, Kansas State University, Mizzou, and the University of Missouri System to develop an expanded regional commitment to cloud biocomputing. Others, including Dr. John Wegman, Head of Computer Science, and Dr. Frank Lee, a Systems Architect, both of IBM, have worked extensively with MU and with the CIOs working through KCALSI to help define a detailed technical vision of what such a cloud biocomputing effort may become in the future.

The development of high-end computing for computational drug discovery, interface data acquisition, management, for bioinformatics of massive amounts of genetic and proteomic data, and applications individually to the advent of personalized medicine, are a current critical missing component of our efforts to develop a Midwest engine in drug discovery. Led by Dr. Gary Allen, the CIOs from Kansas State University, University of Kansas (both their Medical Campus in Kansas City, Kansas and their main campus at Lawrence), Mizzou, and the University of Missouri System have considered the advantages carefully, and they list the following advantages of such high-end computing to the biosciences and health care of the Midwest Region:

- Evaluating hundreds of thousands of molecules to find the most promising candidates for cancer therapies,

- Analyzing huge data sets that are derived from modern medical and biological imaging technologies, and medical records,

- Modeling how complex biological systems operate and how changes in these systems result in human and animal disease,

- Understanding how the uniqueness of an individual's genetic code can be used to optimize medical treatments through personalized medicine, and

- Developing designs for nanotechnology-based health products ranging from microscopic disease screening laboratories to new formulations for the formulating and delivering therapeutic agents.

A proposal request for funding is being prepared by many major research universities in Kansas and Missouri. Hopefully this Great Plains Cloud Computing effort will fill this regional need.

\section{Shared Core Facilities}

The chief research officers of all eleven institutions of higher education, and others from major research nonprofits and corporations that attended the Life Sciences Summit in Kansas City in March, 2010, met to address what will 
be needed to realize the emergence of the Midwest Region. We agreed at that meeting to convene a meeting of the Core Research Facility Managers from each of our Institutions. Dr. Mark McIntosh, Director of the MU Core Facilities and Chair of the Department of Immunology in the Medical School, will coordinate this meeting in the Kansas City area this Fall. The intention of this meeting will be to determine what core facilities may be shared between institutions, and what facilities may be made available to small start-up companies on a cost recovery basis to make the Midwest Region more attractive to companies in the biosciences that are considering locating in this Region.

Currently MU supports a fully functional Core research capability that is available to enhance the research activities of all our investigators, and many of which are available to collaborative researchers across the state and throughout the world. Some of these core facilities report directly to the Office of Research at MU, and they are documented in detail through web links at: http://research.missouri.edu/division/cores.

$\underline{\mathrm{htm}}$. Note that the $800 \mathrm{MHz}$ Nuclear Magnetic Resonance instrument, which is used to infer molecular structure, is unique within the State of Missouri. Other outstanding Core capabilities are located within our Interdisciplinary Research Centers, reporting to the Office of Research through their respective Centers (please see: http://research.missouri.edu/division/center s.htm). Many of these core capabilities are unique within Missouri, including the activation and neutron scattering services through MURR (our research reactor at $\mathrm{MU})$, a state-of-the-art mass spectrometer and x-ray diffractometer at the International Institute for Nano and Molecular Medicine (note: we also have mass spectrometers in Proteomics and X-ray diffraction in the Structural Biology Core, as well as access to the Advanced Light Source synchrotron at Berkeley), and state-of-the-art in vivo imaging facilities for tiny blood vessels and other microscopic tissue at the Dalton Cardiovascular Research Center and for whole animal imaging at the VA Biomolecular Imaging Center. The Bond Life Sciences Center has developed many new capabilities, including a laser-based opto-photonic system that can detect a single metastasized melanoma cancer cell in blood serum, and a new application of an existing compound that is literally thousands of time more effective at treating AIDS than other compounds in the same primate that was responsible for the cross-over infection in humans. The Regional Biocontainment Laboratory (RBL) provides unique facilities for inhalation toxicology studies in laboratory animals, and in imaging laboratory animals in a Biosafety Level 3 Facility. The Interdisciplinary Plant Group (IPG) at MU is among the very best in the world, with thirteen of their faculty members having been elevated to Fellow of the AAAS. This IPG is responsible for the first sequence of the soybean genome, and derived technology from this advance is being used to develop new strains of soybeans and other crops that are resistant to drought and parasites, which increase the value of our harvests by billions of dollars per year. The Transgenic Animal Core Facility and three NIH-funded Animal Resource Centers (Mutant Mouse, Rat and Swine) 
at MU are world-class, and supply knock-out genetic technology to researchers throughout the world who need an animal model that is closer to human where it counts in the pursuit of the development of therapies for human diseases, such as the swine cystic fibrosis model.

Other unique research Core facilities and capabilities exist throughout the State of Missouri: The Missouri University of Science and Technology (MS\&T) in Rolla operates the state's only gallium focused ion beam device, which is able to cut and shape materials at the atomic level. MS\&T also hosts an outstanding capability for analyzing and optimizing the design of the electric power grid across the United States. UMKC has world-class programs in both stroke prevention and treatment, and in the study and development of mineralized tissues for dentistry and orthopedic surgery. Both the Midwest Research Institute in Kansas City, and the Danforth Plant Sciences Center in St. Louis, possess unique capabilities in harvesting oil from microscopic algae, which promises to be an outstanding new source of green energy. Other industries, ranging from Honeywell and Black and Veech in Kansas City, to Monsanto and Boeing in St. Louis, continuously develop new commercial technologies in collaboration with MU that advance the human condition.

MU has led the way in encouraging a broad sharing of our core capabilities across Missouri. MU currently provides genetic sequencing support for several Missouri academic institutions including Washington University in St. Louis, Missouri State University in
Springfield and Truman State University in Kirksville, and flow cytometry core support for Kansas University's Medical Center in Kansas City, Kansas. MU's transgenic cores provide research animal technology throughout the world. MURR provides more radioisotopes for medical applications than any other reactor within the USA, and many companies, such as $\mathrm{ABC}$ Laboratories and those in the MU Life Sciences Incubator, routinely use the MU Core facilities on a full cost recovery basis. Project: Mizzou, which is a collaboration between seven major administrative offices at $\mathrm{MU}$, has been developing a plan to permit these core facilities to be utilized broadly by outside organizations, including businesses, non-profits, and other universities. The Life Sciences Summit, led by MU and hosted by the UMKC campus, set the stage for broad regional collaborations across many states, and initiated the Midwest Bio-Innovation Belt.

In addition, a commercial entity called ABC Labs currently conducts NMR-based molecular stability measurements within the MU core facilities, and they are working closely with MU to expand this core utilization to proteomics and possibly other applications in the future. It is important to note that these collaborations with ABC Labs has helped MU substantially in the development of Good Laboratory Practice (GLP) and Good Manufacturing Practice (GMP) standards within the MU Research Reactor (MURR) core facilities at MU. These standards of GMP and GLP are essential to industry in order to support their efforts to secure intellectual property, and the 
establishment of these very demanding standards within our academic cores, provide an opportunity for excellent experiential learning for our students who aspire to move into commercial laboratory research following their degree awards. Such efforts within our cores provide MU with a unique advantage in our quest to engage more substantially with industry. These GLP / GMP standards require roughly one scientist-year of dedicated effort to establish for each core capability, so any university laboratory that makes such an investment should have a long-term commitment to industrial collaborative research to assure a recovery of this extensive investment. MU's investment in these commercial laboratory standards are a central part of our commitment to fee-for-service work arrangements with industry, which in turn makes MU and the Midwest Region much more attractive for smaller startup biotechnology companies, as well as to more established firms such as ABC Labs.

\section{Conclusions, and Next Steps}

Once a substantial capability in both bioscience cloud computing and in shared core laboratory facilities are established within the Midwest Region, the next step will be to get the leading investigators who are active in drug discovery at our various institutions and at KCALSI to meet at each other's locations with a very concentrated focus on how we can specifically build on each institutions' strengths to make the Midwest Region more competitive than other locations across the United States for all aspects of drug discovery. Once such a general agreement is in place between the participating institutions, then the next step will be to take a comprehensive approach to define the Midwest Region as the optimal location for major drug discovery. The close collaborations that emerge from this effort will likely lead to new regional opportunities for additional work beyond drug discovery. 